\title{
Angular and Frequency-Dependent Wave Velocity and Attenuation in Fractured Porous Media
}

\author{
José M. Carcione, ${ }^{1}$ Boris Gurevich, ${ }^{2,3}$ Juan E. Santos, ${ }^{4,5,6}$ and Stefano Picotti ${ }^{1}$
}

\begin{abstract}
Wave-induced fluid flow generates a dominant attenuation mechanism in porous media. It consists of energy loss due to P-wave conversion to Biot (diffusive) modes at mesoscopicscale inhomogeneities. Fractured poroelastic media show significant attenuation and velocity dispersion due to this mechanism. The theory has first been developed for the symmetry axis of the equivalent transversely isotropic (TI) medium corresponding to a poroelastic medium containing planar fractures. In this work, we consider the theory for all propagation angles by obtaining the five complex and frequency-dependent stiffnesses of the equivalent TI medium as a function of frequency. We assume that the flow direction is perpendicular to the layering plane and is independent of the loading direction. As a consequence, the behaviour of the medium can be described by a single relaxation function. We first consider the limiting case of an open (highly permeable) fracture of negligible thickness. We then compute the associated wave velocities and quality factors as a function of the propagation direction (phase and ray angles) and frequency. The location of the relaxation peak depends on the distance between fractures (the mesoscopic distance), viscosity, permeability and fractures compliances. The flow induced by wave propagation affects the quasishear (qS) wave with levels of attenuation similar to those of the quasi-compressional (qP) wave. On the other hand, a general fracture can be modeled as a sequence of poroelastic layers, where one of the layers is very thin. Modeling fractures of different thickness filled with $\mathrm{CO}_{2}$ embedded in a background medium saturated with a stiffer fluid also shows considerable attenuation and velocity dispersion. If the fracture and background frames are the same, the equivalent medium is isotropic, but strong wave anisotropy occurs in the case of a frameless and highly permeable fracture material, for instance a suspension of solid particles in the fluid.
\end{abstract}

1 Istituto Nazionale di Oceanografia e di Geofisica Sperimentale (OGS), Borgo Grotta Gigante 42c, 34010 Sgonico, Trieste, Italy. E-mail: jcarcione@inogs.it

2 Department of Explorations Geophysics, Curtin University, GPO Box U1987, Perth, WA 6845, Australia. E-mail: B.Gurevich@curtin.edu.au

3 CSIRO Earth Science and Resource Engineering, ARRC, 26 Dick Perry Avenue, Kensington, Perth, WA 6151, Australia.

4 CONICET, Instituto del Gas y del Petróleo, Facultad de Ingeniería, Universidad de Buenos Aires, C1127AAR Buenos Aires, Argentina. E-mail: santos@math.purdue.edu

5 Department of Mathematics, Purdue University, West Lafayette, IN 47907-2067, USA.

6 Universidad Nacional de La Plata, La Plata, Argentina.
Key words: Fractures, anisotropy, attenuation, boundary conditions.

\section{Introduction}

The acoustic characterization of fractures and cracks is important from the point of view of reservoir development (e.g., GuREvich et al., 2009). Moreover, it is also important in $\mathrm{CO}_{2}$ storage to monitor the injected plumes as faults and fractures are generated, where the gas can leak to the surface (Рісотті et al., 2012). A dense set of planar fractures behaves as an effective long-wavelength TI medium, leading to azimuthally varying velocity and attenuation of seismic waves. One of the important mechanisms of seismic attenuation in porous media is wave-induced fluid flow, by which the fast $P$ wave is converted to slow (diffusive) modes of the Biot type (WhIte et al., 1975; PriDE et al., 2004; CARCIONE and PICOTTI; 2006). The phenomenon has been studied for alternating thin poroelastic layers, along the direction perpendicular to the layer planes (WhITE et al., 1975) at all frequencies and propagation angles (KrziKalla and Müller, 2011; CARCione et al., 2011). The case of planar fractures embedded in a poroelastic background medium is a particular case of the thin layer problem, where one of layers becomes extremely thin and compliant. Alternative models, based on distributions of finite cracks, are given in CHAPMAN (2003) and Galvin and Gurevich (2009). A comprehensive review of the wave-induced fluid-flow loss mechanisms, with a discussion of the related relaxation frequencies, is given in the review paper by MüLLER et al. (2010). Readers can also refer to Mavko et al. (2004) for detailed mathematical expressions of the basic loss mechanisms.

In the case of a solid (non-porous) background, Chichinina et al. (2009) and CARCIONe et al. (2012) obtained analytical solutions for the TI and more general anisotropic cases, respectively, i.e., the 
complex and frequency-dependent stiffness components and corresponding wave velocities and quality factors. Regarding poroelastic media, GUREVICH (2003) and BRAJANOvski et al. (2005) found the lowand high-frequency limit elasticities of the equivalent TI medium under the long-wavelength assumption. Moreover, they obtained the expression of the P-wave modulus for waves propagating normal to the fracture planes as a function of frequency. LAMBERT et al. (2006) validated the theory by numerical simulations using a poroelastic reflectivity algorithm. Du et al. (2011) have developed a similar stress-strain relation for fractured porous media in the low-frequency limit.

To obtain the wave properties as a function of frequency and propagation (phase and ray) angle, we need all the stiffness components of the equivalent TI medium. An heuristic approach has been outlined by LAMBERT et al. (2005), who analyzed the qP wave. A physical interpretation has been given by KRzIKALLA and MüLLER (2011) for layered media, who verified the correctness of the method by comparison to numerical solutions. Further tests for layered media have been performed by CARCIONE et al. (2011). Basically, the approach assumes a 1D character of the fluid pressure equilibration process which generates diffusive modes from the fast $\mathrm{P}$ wave, i.e., the fluid-flow direction is perpendicular to the fracture plane. In the presence of horizontal plane layers, the initial fluid pressure field is independent of the type of excitation, i.e., $\mathrm{P}$ waves traveling horizontally or vertically (mainly compression), or $\mathrm{S}$ waves (shearing) will excite the fluid pressure in such a way as to maintain its distribution. For more general layer geometries, the process may depend on the direction of wave propagation and these assumptions could not be strictly valid. As a consequence, the model considers one relaxation function, corresponding to the symmetry-axis P-wave stiffness. From this relaxation function and the high- and lowfrequency elastic limits of the stiffness tensor, we obtain the five stiffnesses of the equivalent medium. We then obtain the quality factors and wave velocities as a function of frequency and propagation angle.

The examples consider a fractured-sandstone model with parameters mainly taken from BRAJANOVSKI et al. (2005), i.e., the properties of the background porous medium and the normal and tangential fracture compliances. Generally, in this work, fractures are planar, rotationally symmetric, highly permeable and have infinite extent. The degree of attenuation and velocity dispersion caused by different types of heterogeneities in the rock properties, namely, porosity, grain and frame moduli, permeability, and fluid properties were studied in detail in CARCIONE and PICOTTI (2006). Here, we focus on the anisotropy properties of wave velocity and attenuation.

\section{Highly Permeable Fracture}

Let us consider a set of fractures parallel to the $(x, y)$-plane in an isotropic porous medium of porosity $\phi$, separated by an average distance $L$, and denote by $Z_{N}$ and $Z_{T}$ the normal and shear excess fracture compliances. Here, we assume that the fracture thickness is nil (extremely small) and that the porosity within the fracture is one, i.e., there is no fracture frame. Moreover, let us denote by $K_{m}$ and $\mu$ the dry-rock bulk and shear moduli of the background porous medium, respectively, with $E_{m}=K_{m}+4 \mu / 3$ and $\lambda_{m}=K_{m}-$ $2 \mu / 3$. Let $K_{s}$ and $K_{f}$ be the grain and fluid moduli, respectively. Biot's effective stress coefficient is

$$
\alpha=1-\frac{K_{m}}{K_{s}} .
$$

Gurevich (2003) and Brajanovski et al. (2005) obtained the low-frequency limit (relaxed) elasticities of the equivalent TI medium,

$$
\begin{aligned}
& c_{11}^{r}=\frac{c_{13}^{r}{ }^{2}}{c_{33}^{r}}+4\left[(1-\gamma) \mu+\frac{\alpha^{2} \gamma^{2} \beta}{1+Z_{N} \beta}\right], \\
& c_{13}^{r}=c_{33}^{r}\left[1-2 \gamma+2 \alpha \gamma \frac{M}{E_{G}} \frac{\alpha+Z_{N} E_{m}}{1+Z_{N} \beta}\right], \\
& c_{33}^{r}=E_{G}\left[1+\frac{Z_{N}\left(\alpha M-E_{G}\right)^{2}}{E_{G}\left(1+Z_{N} \beta\right)}\right]^{-1}, \\
& c_{55}^{r}=\left(\mu^{-1}+Z_{T}\right)^{-1}, \\
& c_{66}^{r}=\mu,
\end{aligned}
$$

where

$$
\begin{gathered}
E_{G}=K_{m}+\alpha^{2} M+\frac{4}{3} \mu, \quad \beta=M E_{m} / E_{G}, \\
\gamma=\mu / E_{m} .
\end{gathered}
$$

(The first Eq. (35) in BrajanovsKi et al. (2005) should be $\left(\mu_{b}^{-1}+Z_{T}\right)^{-1}$ instead of $\mu_{b}^{-1}+Z_{T}$, using their notation). 
The high-frequency limit elasticities (unrelaxed) are given by (BRAJANOVSKI et al., 2005),

$$
\begin{aligned}
& c_{11}=c_{33}=E_{G}, \\
& c_{13}=c_{11}-2 \mu, \\
& c_{55}=c_{55}^{r}, \\
& c_{66}=\mu,
\end{aligned}
$$

where

$$
M=\frac{K_{s}}{1-\phi-K_{m} / K_{s}+\phi K_{s} / K_{f}} .
$$

Following the approach used by KRZIKALLA and MüLLER (2011) for a layered porous medium (see also CARCIONE et al., 2011), we obtain the five stiffnesses of the equivalent TI medium as

$$
p_{I J}(\omega)=c_{I J}+\left(\frac{c_{I J}-c_{I J}^{r}}{c_{33}-c_{33}^{r}}\right)\left[p_{33}(\omega)-c_{33}\right],
$$

where $\omega$ is the angular frequency, and

$$
\begin{gathered}
p_{33}(\omega)=\left[\frac{1}{E_{G}}+\frac{\left(\left(\alpha M / E_{G}\right)-1\right)^{2}}{\beta \vartheta \cot \vartheta+Z_{N}^{-1}}\right]^{-1}, \\
\vartheta=\sqrt{\frac{\omega}{\mathrm{i} D} \frac{L}{2}}, \quad D=\frac{\kappa \beta}{\eta}
\end{gathered}
$$

(Brajanovski et al, 2005), where $\eta$ is the fluid viscosity, $\kappa$ is the permeability and $\mathrm{i}=\sqrt{-1}$. (Note an omission in Eq. (15) of BRAJANOVSKI et al. (2005): Using their notation, $M_{b} / C_{b}$ should be replaced by $L_{b} M_{b} / C_{b}$ in the denominator. Eq. (8) in GuREvicH et al. (2009) is correct). We have used the opposite sign convention to express the properties in the Fourier domain, i.e., $\omega$ has been replaced by $-\omega$ in Eq. (15) of Brajanovski et al. (2005) and Eq. (8) of Gurevich et al. (2009). The quantity $\omega L^{2} /(4 D)$ is the normalized frequency. Equations (6) and (7) are deduced from Eqs. (25), (41) and (42) of Krzikalla and MülLER (2011). Equation (6) is obtained by considering the 1D character of the fluid pressure equilibration process between the background porous medium and the fractures, assuming that the fluid-flow direction is perpendicular to the fracture plane. As a consequence, the model considers one relaxation function, corresponding to the symmetryaxis P-wave stiffness. Therefore, knowing this relaxation function and the high- and low-frequency elastic limits of the stiffness tensor, one can obtain the five complex and frequency-dependent stiffnesses of the equivalent viscoelastic medium. The equations hold for frequencies $\omega \ll \omega_{B}=\eta \pi /$ $\left(\kappa \rho_{f}\right)$, where $\rho_{f}$ is the fluid density, so that the fluid flow in the pores is of Poiseuille type and the effective medium approximation (Backus averaging) is valid.

The low-frequency regime occurs when pressure has enough time to equilibrate between the background medium and the fractures within a wave period. This happens when the diffusion length $\sqrt{D / \omega}$ (or wavelength of Biot's slow wave) is much larger than the period $L$. High frequency means that the frequency is much less than $\omega_{B}$ but much larger than $D / L^{2}$. In both cases, the wavelength of the pulse must be much larger than the spacing $L$ (e.g., CARCIONE, 2007).

The bulk density is given by

$$
\rho=(1-\phi) \rho_{s}+\phi \rho_{f},
$$

where $\rho_{s}$ is the grain density.

\section{General Fractures}

The equivalent medium corresponding to a set of highly permeable fractures can be obtained as a limit case of a layered medium, where one of the layers is very soft and thin. Equation (7) has been obtained in this manner by BRAJANOvSKi et al. (2005). In this section, we do not consider any approximation, the possibility of having a different fluid saturating the fracture, a finite fracture thickness and a poroelastic medium forming the fracture material. Consider a stack of two thin alternating porous layers of thickness $l_{1}$ and $l_{2}$, such that the period of the stratification is $L=l_{1}+l_{2}$. The complex and frequency dependent stiffness $p_{33}$ is given by

$$
p_{33}=\left[\frac{1}{E_{G}}+\frac{2\left(r_{2}-r_{1}\right)^{2}}{\mathrm{i} \omega\left(l_{1}+l_{2}\right)\left(I_{1}+I_{2}\right)}\right]^{-1}
$$

where

$$
r=\frac{\alpha M}{E_{G}}, \quad I=\frac{\eta}{\kappa a} \operatorname{coth}\left(\frac{a L}{2}\right), \quad a=\sqrt{\frac{\mathrm{i} \omega \eta E_{G}}{\kappa M E_{m}}},
$$


for each single layer (White et al., 1975; NoRRIS, 1993; Carcione and Picotti 2006) [see also Carcione (2007), Eq. (7.400)]. If $\phi_{2} \rightarrow 1$ and $Z_{N}=\lim _{l_{2} \rightarrow 0}$ $\left[l_{2} /\left(L E_{m 2}\right)\right]$, we obtain Eq. (7). A similar limit for the shear compliance $Z_{T}$ is obtained by replacing $E_{m 2}$ with $\mu_{2}$. To use Eq. (9) for a highly permeable fracture, one must consider $l_{2} / L \ll 1, \phi_{2} \approx 1, E_{m 2}=$ $l_{2} /\left(L Z_{N}\right), \quad \mu_{2}=l_{2} /\left(L Z_{T}\right), \alpha_{2} \approx 1$ and $\kappa_{2} \gg 1 \mathrm{D}$.

In the general case (an arbitrarily permeable fracture), we consider $l_{2} / L \ll 1$ and general poroelastic parameters. The equivalent medium is obtained from Eq. (6), where $p_{33}$ is given by Eq. (9), with

$c_{66}^{r}=B_{1}^{*}=\langle\mu\rangle$,

$c_{11}^{r}-2 c_{66}^{r}=c_{12}^{r}=B_{2}^{*}=2\left\langle\frac{\lambda_{m} \mu}{E_{m}}\right\rangle+\left\langle\frac{\lambda_{m}}{E_{m}}\right\rangle^{2}\left\langle\frac{1}{E_{m}}\right\rangle^{-1}+\frac{B_{6}^{* 2}}{B_{8}^{*}}$,

$c_{13}^{r}=B_{3}^{*}=\left\langle\frac{\lambda_{m}}{E_{m}}\right\rangle\left\langle\frac{1}{E_{m}}\right\rangle^{-1}+\frac{B_{6}^{*} B_{7}^{*}}{B_{8}^{*}}$,

$c_{33}^{r}=B_{4}^{*}=\left\langle\frac{1}{E_{m}}\right\rangle^{-1}+\frac{B_{7}^{* 2}}{B_{8}^{*}}$

$=\left[\left\langle\frac{1}{E_{m}}\right\rangle-\left\langle\frac{\alpha}{E_{m}}\right\rangle^{2}\left\langle\frac{E_{G}}{M E_{m}}\right\rangle^{-1}\right]^{-1}$,

$c_{55}^{r}=B_{5}^{*}=\left\langle\mu^{-1}\right\rangle^{-1}$,

$B_{6}^{*}=-B_{8}^{*}\left(2\left\langle\frac{\alpha \mu}{E_{m}}\right\rangle+\left\langle\frac{\alpha}{E_{m}}\right\rangle\left\langle\frac{\lambda_{m}}{E_{m}}\right\rangle\left\langle\frac{1}{E_{m}}\right\rangle^{-1}\right)$,

$B_{7}^{*}=-B_{8}^{*}\left\langle\frac{\alpha}{E_{m}}\right\rangle\left\langle\frac{1}{E_{m}}\right\rangle^{-1}$,

$B_{8}^{*}=\left[\left\langle\frac{1}{M}\right\rangle+\left\langle\frac{\alpha^{2}}{E_{m}}\right\rangle-\left\langle\frac{\alpha}{E_{m}}\right\rangle^{2}\left\langle\frac{1}{E_{m}}\right\rangle^{-1}\right]^{-1}$,

and

$$
\begin{aligned}
c_{66} & =c_{66}^{r}, \\
c_{11} & -2 c_{66}=c_{12} \\
& =2\left\langle\frac{\left(E_{G}-2 \mu\right) \mu}{E_{G}}\right\rangle+\left\langle\frac{E_{G}-2 \mu}{E_{G}}\right\rangle^{2}\left\langle\frac{1}{E_{G}}\right\rangle^{-1}, \\
c_{13} & =\left\langle\frac{E_{G}-2 \mu}{E_{G}}\right\rangle\left\langle\frac{1}{E_{G}}\right\rangle^{-1}, \\
c_{33} & =\left\langle\frac{1}{E_{G}}\right\rangle^{-1}, \\
c_{55} & =c_{55}^{r}
\end{aligned}
$$

where we kept the notation of GELINSKY and SHAPIRO (1997) for reference (see also CARCIONE et al. 2011), and where $\langle\varphi\rangle=\left(l_{1} \varphi_{1}+l_{2} \varphi_{2}\right) / L$.
Appendix shows how to obtain the energy velocities and dissipation factors as a function of the ray and phase angles from the complex stiffnesses (6) and the composite density (8).

\section{Example}

The examples consider brine- and oil-saturated sandstones, with grain properties: $K_{s}=37 \mathrm{GPa}, \mu_{s}=$ $44 \mathrm{GPa}$ and $\rho_{s}=2,650 \mathrm{~kg} / \mathrm{m}^{3}$. Brine has the properties: $K_{f}=2.25 \mathrm{GPa}, \rho_{f}=1,040 \mathrm{~kg} / \mathrm{m}^{3}, \eta=0.0018 \mathrm{cP}$, while those of oil are $K_{f}=2 \mathrm{GPa}, \rho_{f}=870 \mathrm{~kg} / \mathrm{m}^{3}, \eta=$ $0.3 \mathrm{cP}$. The dry-rock bulk and shear moduli are given by the Krief model,

$$
\frac{K_{m}}{K_{s}}=\frac{\mu}{\mu_{s}}=(1-\phi)^{3 /(1-\phi)}
$$

(Krief et al., 1990; BRAJANOvski et al., 2005). Porosity and permeability are related by an equation derived by CARCIONE et al. (2000),

$$
\kappa=\frac{r_{g}^{2} \phi^{3}}{45(1-\phi)^{2}},
$$

where $r_{g}=20 \mu \mathrm{m}$ denotes the average radius of the grains.

We consider two sets of compliances taken from BRAJANOVSKI et al. (2005), obtained as

$Z_{N}=\frac{1}{E_{m}}\left(\frac{1}{\Delta N}-1\right)^{-1}$ and $Z_{T}=\frac{1}{\mu}\left(\frac{1}{\Delta T}-1\right)^{-1}$,

where $(\Delta N, \Delta T)=(0.2,0.5)$, giving $1 / Z_{N}=121 \mathrm{GPa}$ and $1 / Z_{T}=14 \mathrm{GPa}$, and $(\Delta N, \Delta T)=(0.02,0.05)$, giving $1 / Z_{N}=1,483 \mathrm{GPa}$ and $1 / Z_{T}=264 \mathrm{GPa}$. In the second set, the two faces of a single fracture are in better contact, since perfect bonding or absence of fractures occurs when $Z_{N} \rightarrow 0$ and $Z_{T} \rightarrow 0$. The quantities $\Delta N$ and $\Delta T$ are dimensionless fracture weaknesses (see Hsu and Schoenberg, 1993; BAKUlin et al., 2000).

First, we assume $\phi=0.25, L=0.2 \mathrm{~m}$ and $\Delta N=$ 0.2 , and compute the P-wave phase velocity and dissipation factor along the direction perpendicular to the fracture plane (see Fig. 1). As can be seen, the relaxation peak for oil is located at the seismic frequency band, while that of brine is located at the sonic band. The peak relaxation frequency is proportional to $\kappa / \eta$ [see Eq. (7.401) in CARCIONE (2007)] 
and can substantially be affected by the normal fracture compliance, as shown in Fig. 2, where the Pwave peak frequency and peak dissipation factor for oil saturation are represented as a function of $\Delta N$. The peak moves from the sonic band for $\Delta N=0.02$ (4 $\mathrm{kHz}$ and $Q=286$ ) to the seismic band for $\Delta N=0.2$ (35 kHz and $Q=26$ ).

Next, we consider oil saturation, $\Delta T=0.5$ and use Eq. (6) to obtain the complex stiffnesses at $35 \mathrm{~Hz}$, where the peak quality factor has a value of 26 . We obtain

$$
\begin{aligned}
& p_{11}=(33.68,0.001), \\
& p_{13}=(5.75,0.034), \\
& p_{33}=(30.71,1.17), \\
& p_{55}=6.96, \\
& p_{66}=13.92
\end{aligned}
$$
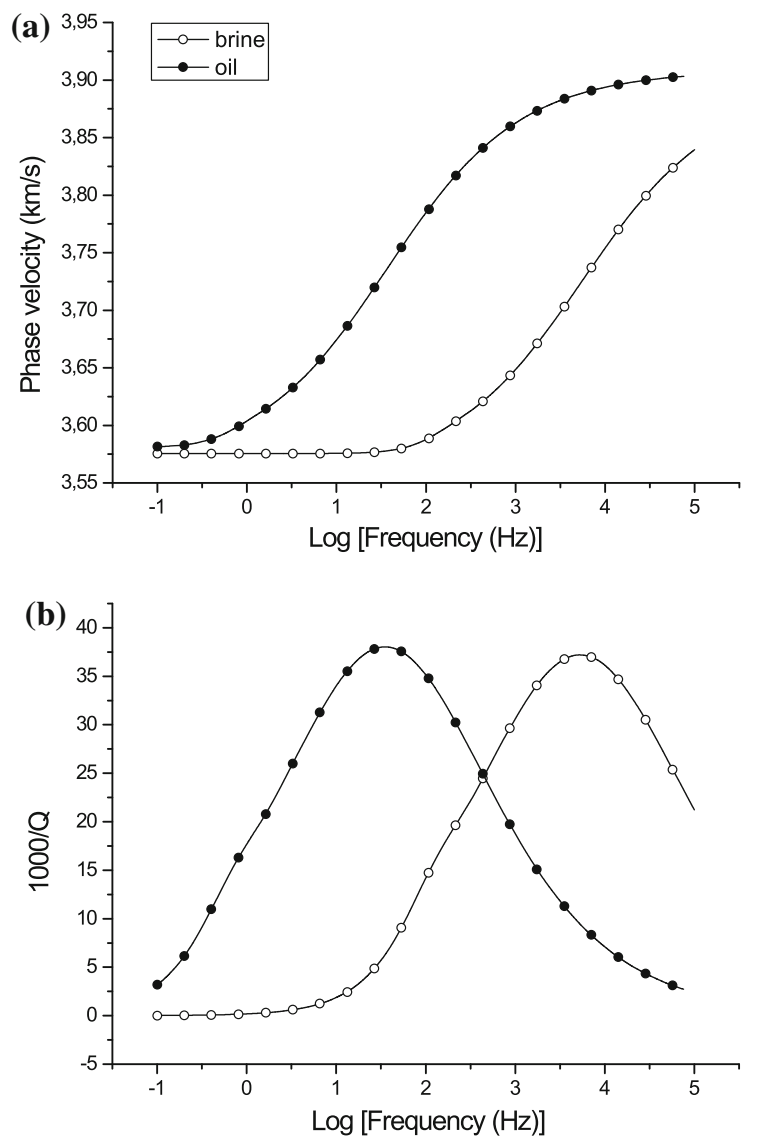

Figure 1

P-wave phase velocity (a) and dissipation factor (b) along the direction perpendicular to the fracture planes for brine and oil filling the pore space with $\Delta N=0.2$ in GPa. The energy velocities and dissipation factors as a function of the ray angle $\psi$ and propagation (phase) angle $\theta$ for oil saturation are represented in Fig. 3a and b, respectively. There is a noticeable shear-wave splitting, also called seismic birefringence, and the coupling between the $\mathrm{qP}$ and $\mathrm{qS}$ waves generates strong shear attenuation $(Q \approx 40)$ at approximately $50^{\circ}$, with magnitudes comparable to those of the qP wave. The $\mathrm{SH}$ wave is lossless. Energy velocity rather than phase velocity is shown, since it represents the wavefront (e.g., CARCIONE, 2007).

The same plots are repeated for oil saturation with $(\Delta N, \Delta T)=(0.02,0.05)$ (Figs. 4,5$).$ In this case, the fracture is stiffer than above, i.e., the two faces of the fracture are in better contact. As can be seen, the peak
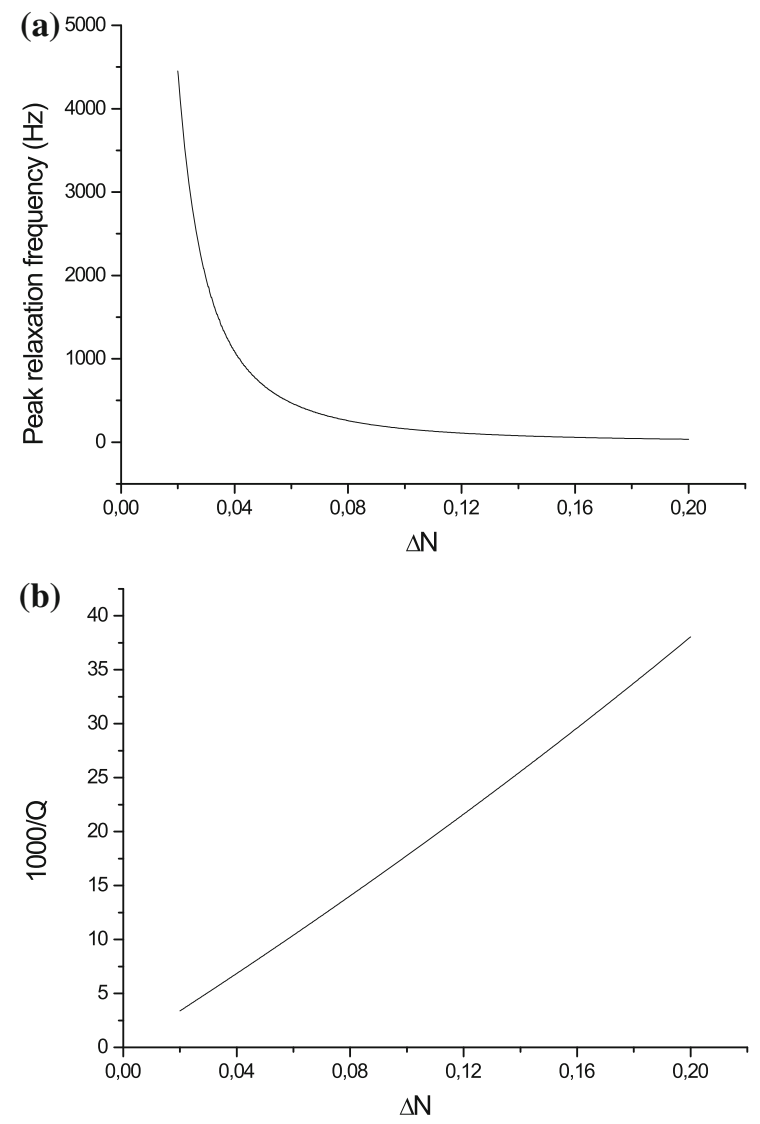

Figure 2

P-wave peak relaxation frequency (a) and peak dissipation factor (b) as a function of the normal fracture parameter $\Delta N$ for oil saturation 

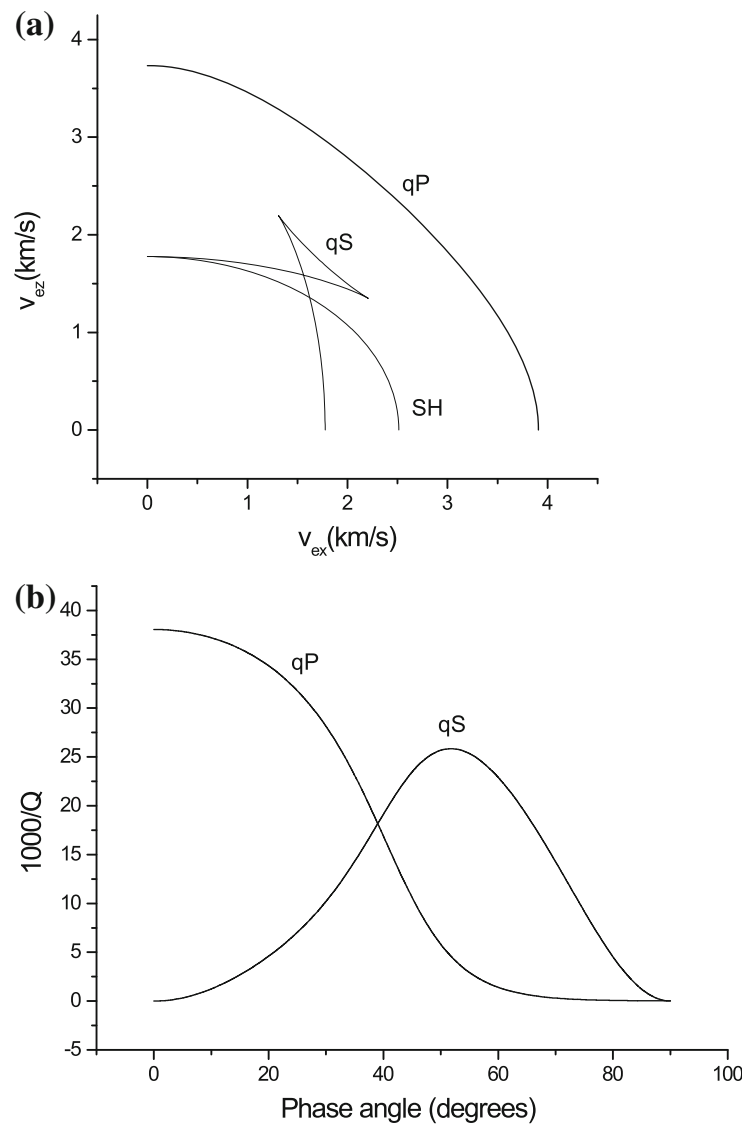

Figure 3

Energy velocity (a) and dissipation factor (b) as a function of the ray and propagation (phase) angles, respectively, for an oil saturated fractured medium with $(\Delta N, \Delta T)=(0.2,0.5)$. The frequency is $35 \mathrm{~Hz}$

has moved to the sonic band and shows less velocity dispersion and attenuation. The P-wave peak quality factor along the vertical direction is equal to 286 . Moreover, the medium is almost isotropic.

Figure 6 represents the dissipation factors for an oil-saturated fractured medium as a function of the ray angle. The $\mathrm{qS}$ factor corresponding to the more compliant fracture set shows triplications (a) as the wavefronts in Fig. 3a, while the stiffer fracture set induces a weak TI medium (b), almost isotropic, with the ray angle approximately equal to the phase angle. Finally, the relaxed and unrelaxed energy velocities (wavefronts) for an oil-saturated fractured medium are shown in Fig. 7, where the range of velocity dispersion can clearly be seen. Major differences occur in the direction normal to the fracture plane
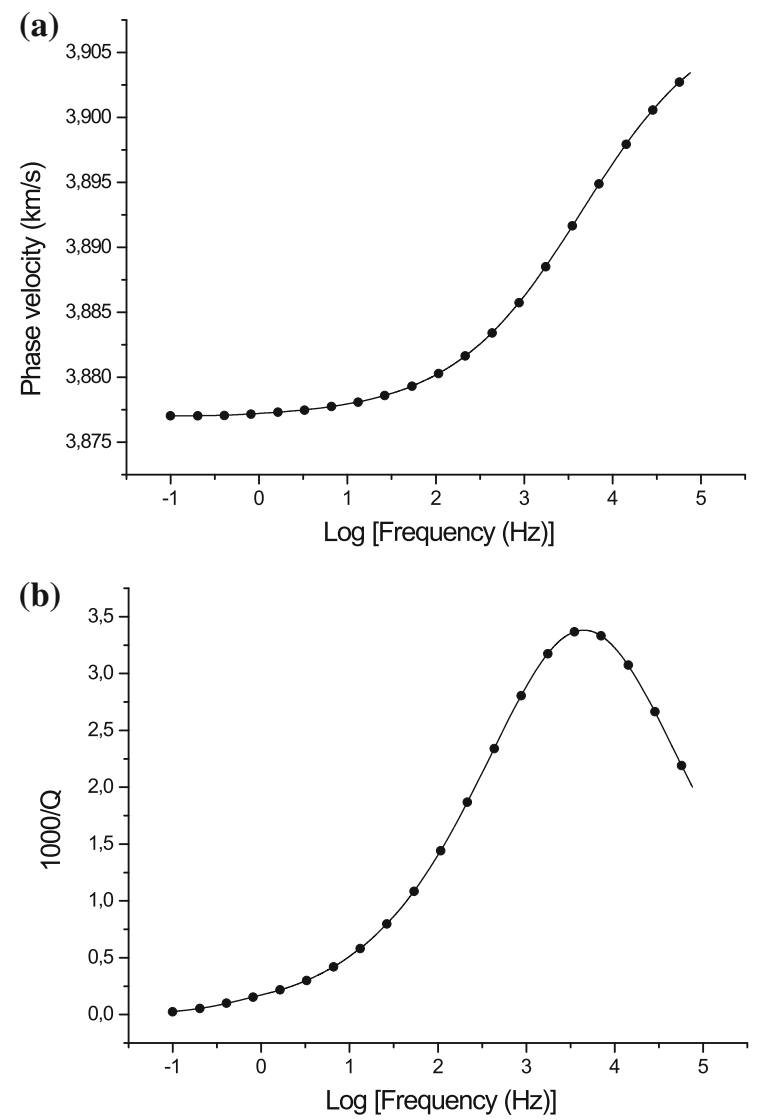

Figure 4

P-wave phase velocity (a) and dissipation factor (b) along the direction perpendicular to the fracture planes for oil filling the pore space $\Delta N=0.02$

(qP wave) and at the location of the cuspidal triangles (qS wave).

Next, we consider a more general case, where the fracture has a finite thickness and is a poroelastic medium. In the first example, the skeleton is the same for both the background medium and fracture material and is given by Eq. (13) with $\phi=0.25$. The fracture is filled with $\mathrm{CO}_{2}$ defined by $K_{f}=0.025 \mathrm{GPa}$, $\rho_{f}=500 \mathrm{~kg} / \mathrm{m}^{3}, \eta=0.00002 \mathrm{cP}$ (CARCIONE et al., 2012). The equivalent medium is obtained from Eqs. (6) and (9)-(12). First, we consider $L=0.2 \mathrm{~m}$ and a fracture thickness $l_{2}=1 \mathrm{~cm}$. Figure 8 shows the phase velocity and dissipation factor for water and oil saturating the background medium. The equivalent medium is isotropic since the shear moduli of the background and fracture media are the same, because the dry- and wet-rock shear moduli are the same 

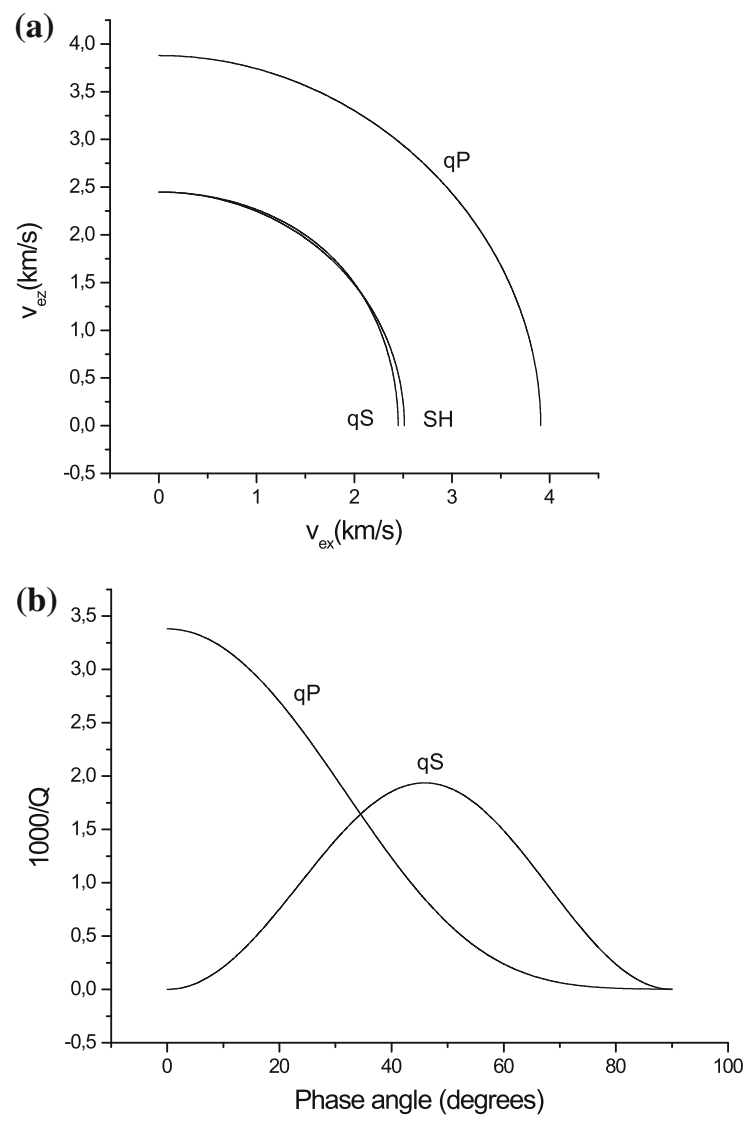

Figure 5

Energy velocity (a) and dissipation factor (b) as a function of the ray and propagation (phase) angles, respectively, for an oilsaturated fractured medium with $(\Delta N, \Delta T)=(0.02,0.05)$. The frequency is $4 \mathrm{KHz}$

according to Gassmann's equations. On the other hand, the effect of fracture thickness $\left(l_{2}=1 \mathrm{~cm}\right.$ and $l_{2}$ $=1 \mathrm{~mm}$ ) is shown in Fig. 9, where the background medium is saturated with brine. As can be seen, varying thickness mainly affects the peak attenuation, while the type of background fluid dictates the location of the relaxation peak.

Finally, we consider a fracture medium with $\phi=$ 0.5 filled with gas in a brine-saturated background $(\phi$ $=0.25$ ), where $L=0.2 \mathrm{~m}$ and $l_{2}=1 \mathrm{~cm}$ (fracture thickness). Since the fracture porosity exceeds the critical porosity, the material is practically a suspension of grains with negligible frame moduli. In this case, the equivalent medium is anisotropic. Figure 10 shows polar plots of the $\mathrm{qP}$ and $\mathrm{qS}$ energy velocities and the dissipation factor as a function of the phase angle. The fact that the background and
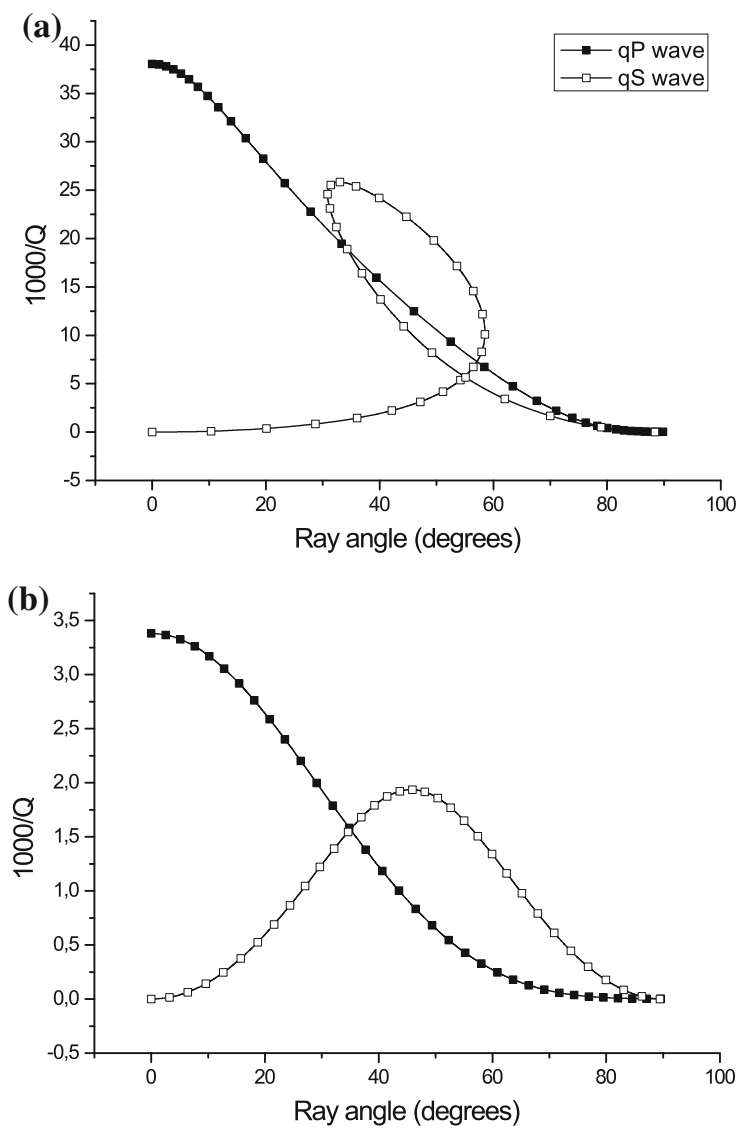

Figure 6

Dissipation factor as a function of the ray angle for an oil-saturated fractured medium. a $(\Delta N, \Delta T)=(0.2,0.5)$ and $f=35 \mathrm{~Hz}$; b $(\Delta N, \Delta T)=(0.02,0.05)$ and $f=4 \mathrm{kHz}$

fracture media have very dissimilar frame moduli induces strong velocity and $Q$ anisotropy. The qP wave shows high dissipation compared to the $\mathrm{qS}$ wave, and the $\mathrm{SH}$ wave is lossless.

The cases presented in Figs. 8, 9 and 10 are more general since the fracture material is explicitly considered as a poroelastic medium, while in the previous examples fractures are modeled by means of fracture weaknesses. Attenuation for both models behaves dissimilarly for the cases considered here. In the general case, the P-wave attenuation is stronger along the fracture planes.

Other cases of interest but showing negligible dissipation involve the presence of a low porosity layers. For instance, if the background medium of the previous example has $\phi=0.05$, we obtain strong anisotropy, with $c_{11}=77 \mathrm{GPa}, c_{33}=22 \mathrm{GPa}, c_{55}=10$ 


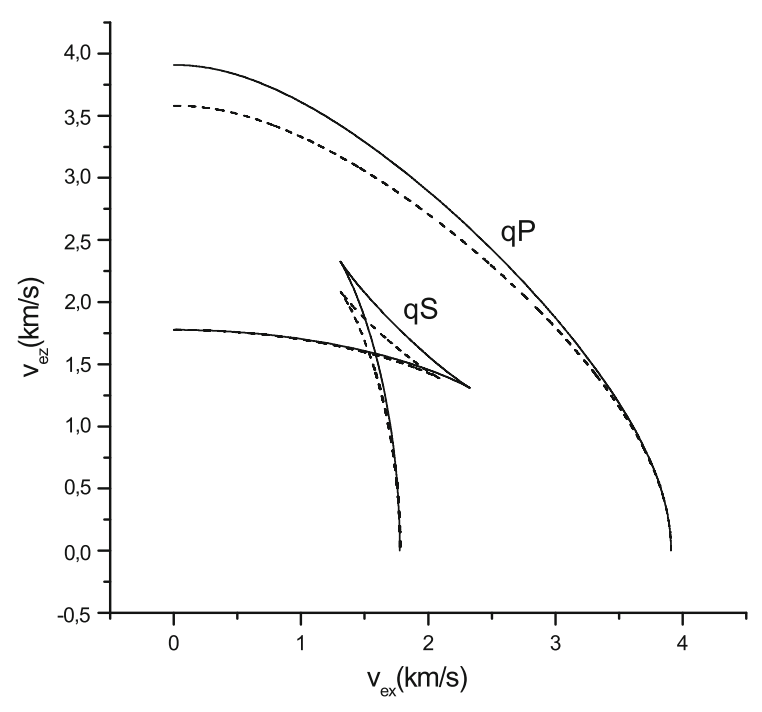

Figure 7

Energy velocities (wavefronts) for an oil-saturated fractured medium, where $(\Delta N, \Delta T)=(0.2,0.5)$ and $f=35 \mathrm{~Hz}$. The dashed and solid lines correspond to the relaxed and unrelaxed states, respectively

GPa and $c_{66}=36 \mathrm{GPa}$. This system could represent a fractured shale. On the other hand, if we consider a fractured medium with $\phi=0.05$ filled with brine (a seal) and a background medium with $\phi=0.25$ filled with $\mathrm{CO}_{2}$, we obtain weak anisotropy.

The implementation of these models in seismic forward modeling in anisotropic porous media to compute synthetic seismograms (e.g., CARCIOnE, 1996; Du et al., 2011) can be important to characterize the seismic response of fractured reservoirs.

\section{Conclusions}

First, we have analyzed the angular propertieswave velocities and dissipation factors-of a fractured medium saturated with a single fluid. The wavelength is much larger than the fracture spacing. Fractures are modeled as boundary discontinuities in the displacement field. The energy velocity and quality factors as a function of the propagation and ray (energy) angles are obtained for homogeneous viscoelastic plane waves (wavenumber and attenuation directions coincide).

The P-wave has strong attenuation along the direction perpendicular to the layering plane, while the attenuation is weak along the fracture planes. The
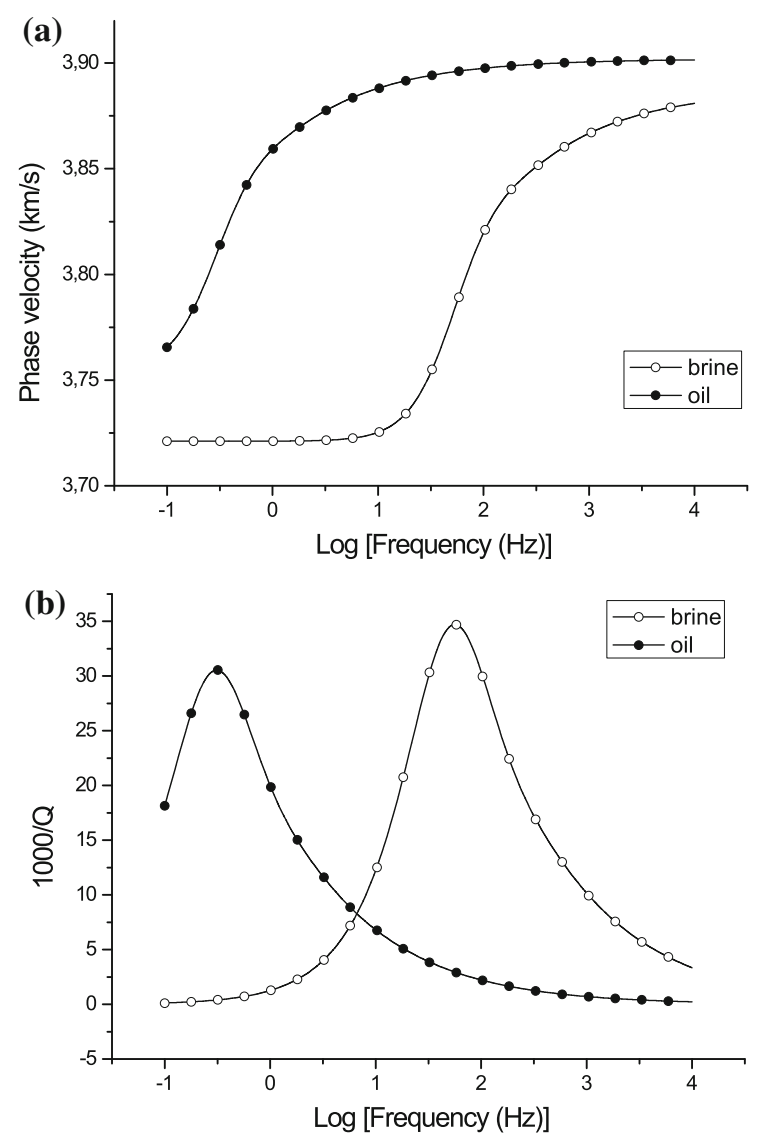

Figure 8

P-wave phase velocity (a) and dissipation factor (b) for $\mathrm{CO}_{2}$ saturated fractures embedded in brine- and oil-saturated media

qS wave has no loss along the directions parallel and perpendicular to the fracture planes and has maximum attenuation at intermediate angles, with magnitudes comparable to those of the $\mathrm{qP}$ wave. Moreover, it shows splitting (triplications) as the wavefronts. On the other hand, the SH wave is lossless. Viscosity (and permeability) dictate the location of the relaxation peak, as well as the fracture spacing and compliances. Higher viscosity (oil vs. brine) implies a lower peak frequency, e.g., for given values of the fracture stiffnesses oil has a peak at the seismic band while brine has a peak at the sonic band, in agreement with the mesoscopic loss mechanism. The fracture spacing has the same effect as the viscosity. Here, the term "mesoscopic" refers to fracture spacing, which is much larger than the pore radius but much smaller than the pulse wavelength. Higher fracture stiffnesses (lower compliances) implies 

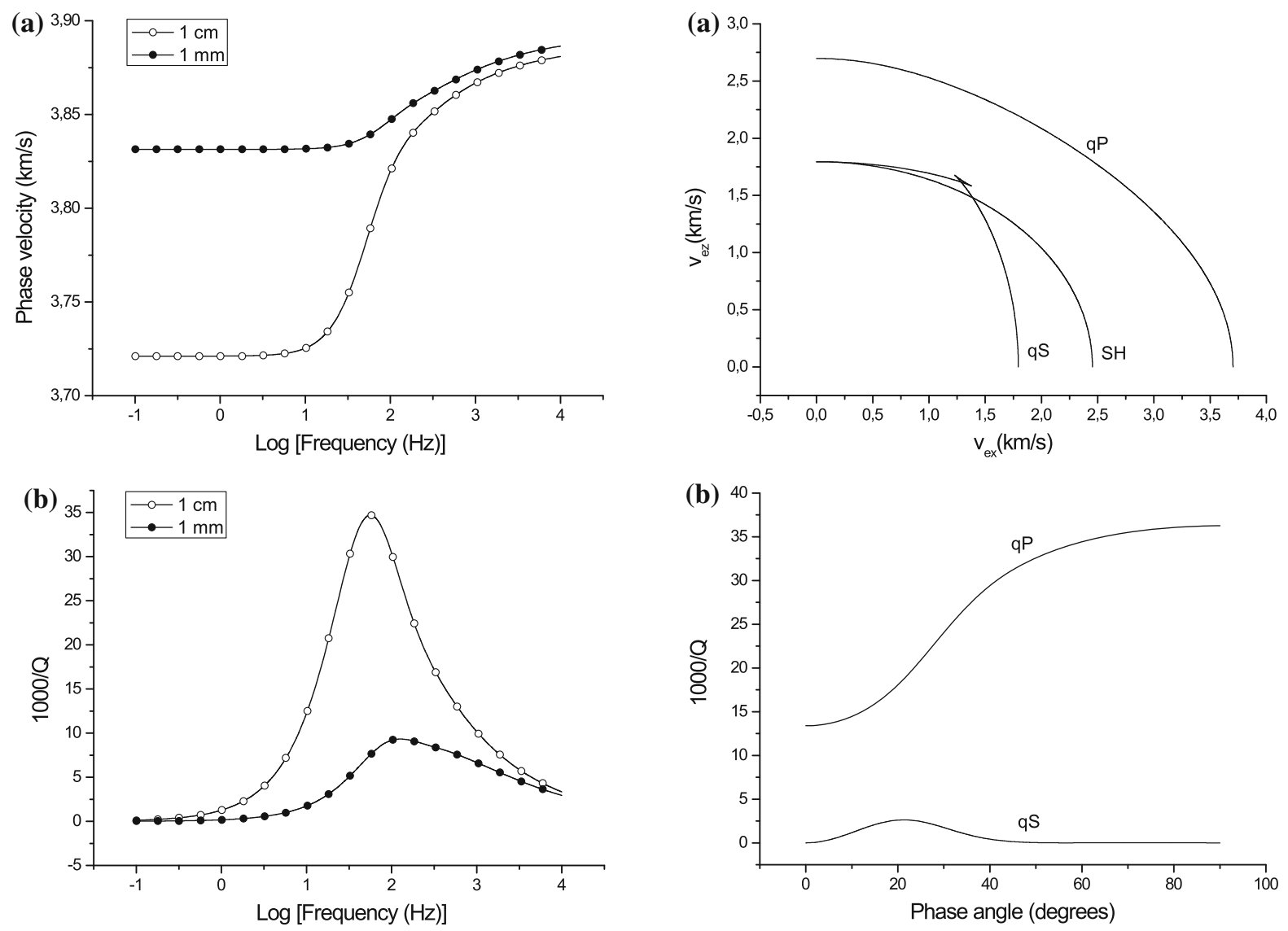

Figure 9

P-wave phase velocity (a) and dissipation factor (b) for $\mathrm{CO}_{2}$ saturated fractures embedded in brine-saturated media. Two fracture thicknesses are considered

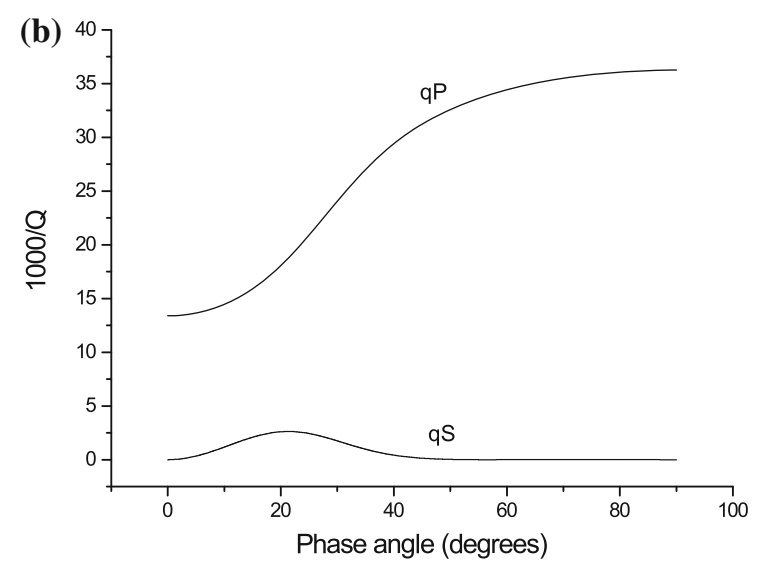

Figure 10

Polar plot of the energy velocity (a) and dissipation factor (b) as a function of the propagation (phase) angle, for finite-thickness fractures $\left(l_{2}=1 \mathrm{~cm}\right)$ saturated with $\mathrm{CO}_{2}$ and embedded in a brinesaturated medium. The fracture period is $20 \mathrm{~cm}$ and the frequency is $50 \mathrm{~Hz}$

lower attenuation and anisotropy and a higher peak relaxation frequency.

Then, we consider the general case, (an arbitrarily permeable fracture of finite thickness). As before, the wavelength is much larger than the fracture spacing. Varying thickness mainly affects the peak attenuation, while the type of background fluid affects the location of the relaxation peak. Strong anisotropy is present if the fracture material has no frame (and high permeability). The presence of low porosity layers inhibits the mesoscopic loss mechanism.

The proposed model can be important in determining the orientation of fractures on the basis of seismic velocity and attenuation measurements.

Moreover, it can be useful to perform sensitivity studies for the detection of $\mathrm{CO}_{2}$ leakages through fractures, related to carbon dioxide storage in depleted reservoirs.

\section{Acknowledgments}

J.E.S was partially funded by PIP 112-200801-00952 (CONICET, Argentina). J.M.C and S.P. have been partially funded by the European Union under the framework of the CO2CARE project. We thank two anonymous reviewers for useful comments. 


\section{Appendix}

\section{Wave Velocities and Quality Factors}

The complex velocities are required to calculate wave velocities and quality factors of the fractured medium. They are given by

$$
\begin{aligned}
v_{\mathrm{qP}} & =(2 \rho)^{-1 / 2} \sqrt{p_{11} l_{1}^{2}+p_{33} l_{3}^{2}+p_{55}+A} \\
v_{\mathrm{qSV}} & =(2 \rho)^{-1 / 2} \sqrt{p_{11} l_{1}^{2}+p_{33} l_{3}^{2}+p_{55}-A} \\
v_{\mathrm{SH}} & =\rho^{-1 / 2} \sqrt{p_{66} l_{1}^{2}+p_{55} l_{3}^{2}} \\
A & =\sqrt{\left[\left(p_{11}-p_{55}\right) l_{1}^{2}+\left(p_{55}-p_{33}\right) l_{3}^{2}\right]^{2}+4\left[\left(p_{13}+p_{55}\right) l_{1} l_{3}\right]^{2}}
\end{aligned}
$$

(CARcione, 2007), where $l_{1}=\sin \theta$ and $l_{3}=\cos \theta$ are the directions cosines, $\theta$ is the propagation angle between the wavenumber vector and the symmetry axis, and the three velocities correspond to the $\mathrm{qP}, \mathrm{qS}$ and $\mathrm{SH}$ waves, respectively. The phase velocity is given by

$$
v_{p}=\left[\operatorname{Re}\left(\frac{1}{v}\right)\right]^{-1},
$$

where $v$ represents either $v_{\mathrm{qP}}, v_{\mathrm{qSV}}$ or $v_{\mathrm{SH}}$. The energy-velocity vector of the $\mathrm{qP}$ and $\mathrm{qSV}$ waves is given by

$$
\frac{\mathbf{v}_{e}}{v_{p}}=\left(l_{1}+l_{3} \cot \psi\right)^{-1} \hat{\mathbf{e}}_{1}+\left(l_{1} \tan \psi+l_{3}\right)^{-1} \hat{\mathbf{e}}_{3}
$$

(CARCIONE, 2007), where

$$
\tan \psi=\frac{\operatorname{Re}\left(\beta^{*} \mathrm{X}+\xi^{*} \mathrm{~W}\right)}{\operatorname{Re}\left(\beta^{*} W+\xi^{*} Z\right)},
$$

defines the angle between the energy-velocity vector and the $z$-axis (the ray angle),

$$
\begin{aligned}
\beta & =\sqrt{A \pm B}, \\
\xi & = \pm \mathrm{pv} \sqrt{A \mp B}, \\
B & =p_{11} l_{1}^{2}-p_{33} l_{3}^{2}+p_{55} \cos 2 \theta,
\end{aligned}
$$

where the upper and lower signs correspond to the $\mathrm{qP}$ and qSV waves, respectively. Moreover,

$$
\begin{aligned}
W & =p_{55}\left(\xi l_{1}+\beta l_{3}\right), \\
X & =\beta p_{11} l_{1}+\xi p_{13} l_{3}, \\
Z & =\beta p_{13} l_{1}+\xi p_{33} l_{3}
\end{aligned}
$$

(CARCIONE, 2007), where "pv" denotes the principal value, which has to chosen according to established criteria.

On the other hand, the energy velocity of the SH wave is

$$
\mathbf{v}_{e}=\frac{v_{p}}{\rho \operatorname{Re}(v)}\left[l_{1} \operatorname{Re}\left(\frac{p_{66}}{v}\right) \hat{\mathbf{e}}_{1}+l_{3} \operatorname{Re}\left(\frac{p_{55}}{v}\right) \hat{\mathbf{e}}_{3}\right] .
$$

Finally, the quality factor is given by

$$
Q=\frac{\operatorname{Re}\left(v^{2}\right)}{\operatorname{Im}\left(v^{2}\right)} .
$$

\section{REFERENCES}

Bakulin A., GrechKa V., Tsvankin I. (2000) Estimation of fracture parameters from reflection seismic data-Part I: HTI model due to a single fracture set. Geophysics 65:1788-1802

Brajanovski M., Gurevich B., Schoenberg M. (2005) A model for $P$-wave attenuation and dispersion in a porous medium permeated by aligned fractures. Geophysical Journal International 163:372-384

CARCIONE J.M. (1996) Wave propagation in anisotropic, saturated porous media: plane wave theory and numerical simulation. Journal of the Acoustical Society of America 99:2655-2666

CArcione, J. M. (2007) Wave fields in real media: Wave propagation in anisotropic, anelastic, porous and electromagnetic media, Handbook of Geophysical Exploration, vol. 38, Elsevier (2nd edition, revised and extended).

Carcione, J. M., Gei, D., Picotti, S., and Michelini, A. (2012), Cross-hole electromagnetic and seismic modeling for CO2 detection and monitoring in a saline aquifer, Journal of Petroleum Science and Engineering 100:162-172, doi:10.1016/ j.petrol.2012.03.018. http://dx.doi.org/10.1016/j.petrol.2012.03.018

Carcione J.M., Gurevich B., Cavallini F. (2000) A generalized Biot-Gassmann model for the acoustic properties of shaley sandstones. Geophys. Prosp 48:539-557

Carcione J.M., Picotti S. (2006) P-Wave seismic attenuation by slow-wave diffusion: Effects of inhomogeneous rock properties. Geophysics 71:O1-O8

Carcione J.M., Santos J.E., Picotti S. (2011) Anisotropic poroelasticity and wave-induced fluid flow. Harmonic finite-element simulations. Geophysical Journal International 186:1245-1254

Carcione, J. M., Santos, J. E., and Picotti, S. (2012), Fractureinduced anisotropic attenuation, Rock Mech. Rock Eng, doi: 10.1007/s00603-012-0237-y.

Chapman M. (2003) Frequency dependent anisotropy due to mesoscale fractures in the presence of equant porosity. Geophysical Prospecting 51:369-379

Chichinina T.I., Obolentseva I.R., Ronquillo-Jarillo G. (2009) Anisotropy of seismic attenuation in fractured media: Theory and ultrasonic experiment. Transport in Porous Media 79:1-14

Du Q.Z., Wang X.M., Ba J., Zhang Q. (2011) An equivalent medium model for wave simulation in fractured porous rocks. Geophysical Prospecting, doi:10.1111/j.1365-2478.2011.01027.x 
Gelinsky S., Shapiro S.A. (1997) Poroelastic backus-averaging for anisotropic, layered fluid and gas saturated sediments. Geophysics 62:1867-1878

Galvin R.J., Gurevich B. (2009) Effective properties of a poroelastic medium containing a distribution of aligned cracks. Journal of Geophysical Research 114:B07305 doi:10.1029/2008 JB006032.

GuREvich B. (2003) Elastic properties of saturated porous rocks with aligned fractures. Journal of Applied Geophysics 54:203218

Gurevich B., Brajanovski M., Galvin R.J., Müller T. M., Toms-Stewart J. (2009) P-wave dispersion and attenuation in fractured and porous reservoirs-poroelasticity approach. Geophysical Prospecting 57:225-237

Hsu C.J., Schoenberg M. (1993) Elastic waves through a simulated fractured medium. Geophysics 58:964-977

Krief M., Garat J., Stellingwerff J., Ventre J. (1990) A petrophysical interpretation using the velocities of $P$ and $S$ waves (full waveform sonic). The log Analyst 31:355-369

Krzikalla, F., and Müller, T. (2011), Anisotropic P-SV-wave dispersion and attenuation due to inter-layer flow in thinly layered porous rocks, Geophysics, 76, WA135; http://dx.doi. org/10.1190/1.3555077.

Lambert, G., Gurevich, B., and Brajanovski, M. (2005), Frequency-dependent anisotropy of porous rocks with aligned fractures, Poromechanics III, Proceedings of 3rd Biot Conference on Poromechanics, May 2005, pp. 309-314.

Lambert G., Gurevich B., Brajanovski M. (2006) Attenuation and dispersion of $P$-waves in porous rocks with planar fractures: Comparison of theory and numerical simulations. Geophysics 71:N41-N45

Mavko, G., MukerJi, T., and Dvorkin, J. (2004), The Rock Physics Handbook, Cambridge University Press.

Müller T., Gurevich B., Lebedev M. (2010) Seismic wave attenuation and dispersion resulting from wave-induced flow in porous rocks-a review. Geophysics 75:A147-A164

NorRIS A.N. (1993) Low-frequency dispersion and attenuation in partially saturated rocks. J. Acoust. Soc. Am 94:359-370

Picotti, S., Carcione, J. M., Gei, D., Rossi, G., and Santos, J. E. (2012), Seismic modeling to monitor CO2 geological storage: The Atzbach-Schwanenstadt gas field, J. Geophys. Res., 117, B06103, 18 PP., doi:10.1029/2011JB008540.

Pride S.R., Berryman J.G., Harris J.M. (2004) Seismic attenuation due to wave-induced flow. J. Geophys. Res 109:B01201 doi: $10.1029 / 2003$ JB002639.

White J.E., Mikhaylova N.G., Lyakhovitskiy F.M. (1975) Low-frequency seismic waves in fluid saturated layered rocks. Izvestija Academy of Sciences USSR, Phys. Solid Earth 11:654-659

(Received October 1, 2012, revised December 5, 2012, accepted December 11, 2012, Published online February 9, 2013) 